\title{
Making Nutritional Blocks Based on Scirpus rigidus (Totorilla) Flour for the Feeding and Fattening of Guinea Pigs
}

\section{Elaboración de Bloques Nutricionales a Base de Harina de Scirpus Rigidus (Totorilla) para la Alimentación de Cuyes en Crecimiento y Engorde}

I International Seminar of Livestock and Agroindustrial Production ESPOCH 2020

Corresponding Author:

J. E. Usca Méndez

juscamendez@yahoo.es

Published: 2 September 2021

Production and Hosting by

Knowledge E

(c) N. M. Cusquillo Quispillo et al. This article is distributed under the terms of the Creative Commons Attribution License, which permits unrestricted use and redistribution provided that the original author and source are credited.
N. M. Cusquillo Quispillo1, J. E. Usca Méndez¹, I. P. Salgado Tello1, and A. M. Castillo Reinoso ${ }^{2}$

${ }^{1}$ Facultad de Ciencias Pecuarias, Escuela Superior Politécnica de Chimborazo, Riobamba, Ecuador

${ }^{2}$ Facultad de Ciencias, Escuela Superior Politécnica de Chimborazo, Riobamba, Ecuador

\section{Abstract}

The present study was carried out in the Guano Canton, San José de Chocón community. The objective was to evaluate the productive behavior of guinea pigs when totorilla flour (Scirpus rigidus) is used in their daily feeding. A randomized design was used in a two-factor combinatorial arrangement, for Factor A (flour levels) and Factor B (sex), with four treatments and four repetitions of each versus a control treatment. The treatments were: TO (0\%), T1 (5\%), T2 (10\%), T3 (15\%), and T4 (20\%). The evaluation period was 75 days, with an initial weight of $0.41 \mathrm{~kg}$ (21 days old). No significant difference was found $(p<0.05$ ) for the final weights (PF), weight gain (GP), total food consumption (CTA), or feed conversion (CA). However, significant differences were found in the weight of the carcass (PC) $(p \leq 0.01)$; the highest weight was observed in T2 (10\% of totorilla flour) with $0.80 \mathrm{~kg} \pm 0.02$, and in T0; T3 presented lower results. Significant differences were also found for performance of the carcass (RC) $(p \leq 0.01)$; the higher values were observed in the $\mathrm{T} 4$ and $\mathrm{T} 1$ treatments with $77.63 \%$ and $74.63 \%$, respectively; a lower value was observed in $\mathrm{T} 3$ with $63.82 \%$. In terms of the sex factor variable, male animals presented better results than female ones. $20 \%$ totorilla flour corresponded to USD 1.32 profitability. Based on the results, we can conclude that adding totorilla flour to guinea pig feed does not affect their productive behavior and increases the quality of the livestock. For cuyicultural production, the implementation of totorilla flour in guinea pig feed provides a new food alternative and lowers costs.

Keywords: technology and agricultural sciences, animal science, totorilla, Scirpus rigidus, totorilla flour, feeding in guinea pigs, nutritional block.

\section{Resumen}

El presente estudio se realizó en la comunidad de San José de Chocón, cantón Guano. El objetivo fue evaluar el comportamiento productivo de los cuyes cuando en su alimentación diaria se utiliza harina de totorilla (Scirpus rigidus) en la elaboración de bloques nutricionales. Se utilizó un Diseño Completamente al Azar en arreglo combinatorio de dos factores, para el Factor A (niveles de harina), Factor B (sexo), con 4 tratamientos y 4 repeticiones cada uno 
frente un tratamiento control. Los tratamientos fueron T0 (0\%), T1 (5\%), T2 (10\%), T3 (15\%), T4 (20\%), en un periodo de evaluación de 75 días, con un peso inicial de 0,41 kg (21 días de edad). Los resultados no mostraron diferencias significativas $(p<0,05)$ en las variables, en pesos finales (PF), ganancia de peso (GP), consumo total de alimento (CTA), conversión alimenticia (CA). Sin embargo, se encontró diferencias altamente significativas en peso a la canal (PC), ( $p \leq 0,01)$, siendo el mayor peso para el T2 (10\% de harina de totorilla) con $0,80 \mathrm{~kg} \pm 0,02$, y T0; T3 presentaron resultados más bajos. Rendimiento a la canal (RC) se reportan diferencias altamente significativas $(p \leq 0,01)$, obteniendo los valores más altos para los tratamientos T4; T1; con 77,63; 74,63 (\%) y se observó un menor valor en el T3 con $63,82 \%$. Para el factor sexo los animales machos presentan mejor resultado a diferencia de las hembras. Se consiguió con el empleo de $20 \%$ de harina de totorilla con 1,32 USD rentabilidad. Concluyendo que al adicionar harina de totorilla en la alimentación de cuyes no afecta el comportamiento productivo y aumenta la calidad del semoviente. Para la producción cuyícolas la implementación de harina de totorilla en la alimentación de cobayos permitirá tener nueva alternativa alimenticia y abaratar costos.

Palabras Clave: tecnología y ciencias agropecuarias, zootecnia, Totorilla (Scirpus rigidus), harina de totorilla, alimentación en cuyes, bloque nutricional.

\section{Introducción}

La producción de cuyes en la zona interandina y en la provincia de Chimborazo, en las zonas rurales del cantón Guano. Esta actividad tiene gran importancia ya que genera réditos económicos y permite al campesino aprovechar sus recursos y a la vez disponer de proteína de origen animal para su alimentación [1]. En la actualidad la crianza del cuy se realiza de manera tradicional o empírica, debido a que los organismos encargados de la difusión de tecnologías no lo hacen. El resultado es una explotación deficiente, tanto en calidad como en cantidad [2].

Esta especie animal necesita estar bien alimentado para lograr un eficiente desarrollo; tomando en cuenta que la alimentación representa del $70 \%$ al $80 \%$ de los costos de producción [3]. Una deficiente alimentación da como resultado bajo rendimiento y productividad, mayor susceptibilidad a enfermedades, altos porcentajes de mortalidad, salida de los animales al mercado en un mayor tiempo, resultando como consecuencia una actividad que genera bajos de réditos económicos [4].

La producción de cuy es importante ya que ayuda a otorgar ingresos económicos a las familias del sector rural, por lo cual resulta muy importante para el pequeño productor. Sin embargo, la falta de iniciativas para el uso adecuado de los recursos forrajeros existentes en la zona se constituye en una limitante. Hasta el momento, la falta de información sobre el uso de bloques nutricionales para alimentar cuyes es nula, ya que casi no se emplea a nivel de pequeños productores [5].

Para la elaboración, se puede utilizar insumos alimenticios del medio, se puede lograr un mejor aprovechamiento. Una alternativa para la alimentación de estos animales es la planta de totorilla (Scirpus rigidus), que transformada en harina lo que busca es poder reemplazar a las materias primas tradicionales, ya que la misma aporta nutrientes, 
abarata los costos y constituye en una excelente alternativa en la alimentación animal, ya que se pueda disponer durante todo el año [6]. Con la elaboración del bloque nutricional a base de harina de totorilla, se busca satisfacer las demandas de nutrientes que requieren estos semovientes durante la etapa de crecimiento-engorde y de esta manera poder recomendar la utilización al sector que se dedica a la crianza de cuyes. Con estos antecedentes el presente trabajo de investigación se orientó a: Evaluar el comportamiento productivo de los cuyes cuando en su alimentación diaria se utiliza harina de totorilla en la elaboración de bloques nutricionales. Determinar el nivel más adecuado $(5,10,15,20 \%)$ de la utilización de harina de totorilla en la alimentación de cuyes en crecimiento y engorde. Determinar la calidad de los bloques nutricionales a través del Análisis bromatológico y Determinar los costos de producción de los tratamientos en estudio.

\section{Materiales y Métodos}

\subsection{Localización y duración del experimento}

La presente investigación se desarrollo en la Comunidad San José de Chocón perteneciente al Cantón Guano, ubicado a 14,6 km de Riobamba tomando la vía a llapo. A una altitud de 2620 m.s.n.m. y con una latitud sur $1^{\circ} 35^{\prime} 59,03808^{\prime \prime}$ y una longitud oeste $78^{\circ} 38^{\prime} 8,592$ ". La temperatura media anual es de $14,2^{\circ} \mathrm{C}$, posee una precipitación promedio de $523 \mathrm{~mm} / a$ ño. La presente investigación tuvo una duración de 75 días distribuidos en diferentes actividades como; inicio de la investigación, suministro de raciones alimenticias, limpieza y desinfección de pozas, desparasitación y evaluación.

\subsection{Unidades experimentales}

Para el desarrollo de la presente investigación se utilizaron 80 cuyes (40 machos y 40 hembras) de línea mejorada de 21 días de edad y con un peso promedio de $414 \mathrm{~g}$.

\subsection{Tratamientos y diseño experimental}

Para el desarrollo de la presente investigación se utilizaron 4 tratamientos a base de diferentes niveles de harina de totorilla en bloques nutricionales (5,10, 15 y $20 \%)$ para ser comparado con un tratamiento control. Se aplicó un Diseño Completamente al Azar (DCA) en arreglo combinatorio de dos factores, en donde el factor $A$ está constituido por los niveles de harina de totorilla y el factor $B$ en el sexo, con 4 repeticiones y un tamaño de la unidad experimental fue de 2 animales, es decir 8 animales por sexo y 16 animales para cada uno de los tratamientos.

$$
Y i j k=\mu+\alpha i+\beta j+(\alpha i * \beta j)+\xi i j k,
$$


donde: Yijk: Una observación cualquiera; $\mu$ : Efecto de la media por observación; $\alpha$ i: Efecto del factor $A ; \beta j$ : Efecto del factor $B ; \alpha i{ }^{*} \beta j$ : Efecto de la interacción entre el factor

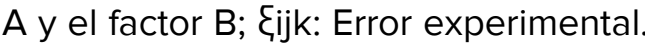

\subsection{Composición de la Ración Experimental para la etapa de crec- imiento y engorde}

\section{Table 1}

Composición de las raciones experimentales (Cusquillo et al., 2019).

\begin{tabular}{|c|c|c|c|c|c|}
\hline \multirow[b]{2}{*}{ Ingredientes } & \multicolumn{5}{|c|}{ Niveles de harina totorilla (\%) } \\
\hline & 0 & 5 & 10 & 15 & 20 \\
\hline Maíz & 9,10 & 12,50 & 15,50 & 18,50 & 27,20 \\
\hline Afrecho trigo & 16,99 & 13,99 & 8,99 & 6,15 & 5,05 \\
\hline Polvillo arroz & 15,76 & 9,66 & 14,36 & 12,00 & 10,00 \\
\hline Afrecho maíz & 21,00 & 20,60 & 13,00 & 10,00 & 0,00 \\
\hline Torta de soya & 23,30 & 24,40 & 24,30 & 24,50 & 23,90 \\
\hline Sal yodada & 0,30 & 0,30 & 0,30 & 0,30 & 0,30 \\
\hline Fosfato di cálcico & 1,00 & 1,00 & 1,00 & 1,00 & 1,00 \\
\hline Carbonato de calcio & 1,00 & 1,00 & 1,00 & 1,00 & 1,00 \\
\hline Premezcla & 0,50 & 0,50 & 0,50 & 0,50 & 0,50 \\
\hline Grasa vegetal & 4,00 & 4,00 & 4,00 & 4,00 & 4,00 \\
\hline Hna. totorilla & 0,00 & 5,00 & 10,00 & 15,00 & 20,00 \\
\hline Antimicótico & 0,05 & 0,05 & 0,05 & 0,05 & 0,05 \\
\hline Cemento & 3,00 & 3,00 & 3,00 & 3,00 & 3,00 \\
\hline Melaza & 4,00 & 4,00 & 4,00 & 4,00 & 4,00 \\
\hline $\begin{array}{l}\text { Costo, } \mathrm{Kg} / \text { Bloque } \\
\text { nutricional }\end{array}$ & 0,43 & 0,42 & 0,41 & 0,41 & 0,40 \\
\hline Total & 100,00 & 100,00 & 100,00 & 100,00 & 100,00 \\
\hline
\end{tabular}

\subsection{Mediciones experimentales}

Las variables a ser evaluadas fueron: peso inicial, $\mathrm{kg}$, peso final, $\mathrm{kg}$, ganancia de peso, $\mathrm{kg}$, consumo de forraje, $\mathrm{kg} \mathrm{MS}$, consumo de bloque, $\mathrm{kg} \mathrm{MS}$, consumo total de alimento, kg MS, conversión alimenticia, peso a la canal, kg, rendimiento a la canal, \%, mortalidad, No., beneficio /costo, \$, y el análisis bromatológico de los bloques nutricionales.

\subsection{Análisis estadísticos y pruebas de significancia}

Los resultados experimentales que se obtuvieron fueron sometidos a los siguientes análisis estadísticos: 
- Análisis de varianza para las diferencias de las medias de los tratamientos (ADEVA).

- Separación de medias según la prueba de Tukey a un nivel de significancia ( $p \leq$ $0,05)$ y $(p \leq 0,01)$

- Análisis de la regresión y correlación (variables que presentaron significancia).

\section{Resultados y Discusión}

\subsection{Evaluación del comportamiento productivo de los cuyes en la etapa crecimiento engorde}

\subsubsection{Peso inicial, $\mathrm{Kg}$}

El peso promedio inicial de los cuyes que se utilizaron para la presente investigación fue de $0,41 \mathrm{~kg}$ de esta manera se inició la experimentación con pesos homogéneos (Tabla 2).

\subsubsection{Peso final, $\mathrm{Kg}$}

Los resultados de peso final de los cuyes al ser sometidos al análisis de varianza no presentaron diferencias estadísticas $(p>0,05)$, por efecto de los tratamientos obteniendo una media T2 (10\% de harina de totorilla) con 1,09 $\mathrm{kg} \pm 0,02$, T0 (0\% de harina de totorilla) con 0,99 $\mathrm{kg} \pm 0,02$ que presenta el valor más bajo (Tabla 2).

En relación a la variable peso final [7], al evaluar el efecto de diferentes niveles de harina de retama más melaza en la elaboración de bloques nutricionales en la alimentación de cuyes donde el mayor peso final registrado fue de 0,972 kg con 0\% de harina de retama más melaza. Así mismo [8], evaluó diferentes niveles de harina de fideo en la alimentación de cuyes en la etapa de crecimiento engorde en relación con la variable peso final obtuvo tratamiento con $30 \%$ de harina con un peso $1,29 \mathrm{~kg}$. En cambio [9] estudió la utilización de bloques nutricionales a base de harina de maralfalfa en la alimentación de cuyes, reportando un peso final de 1,15 kg. Estos resultados probablemente se deban a la calidad nutricional del bloque y forraje suministrado a los animales.

\subsubsection{Ganancia de peso, $\mathrm{Kg}$}

Al analizar la variable ganancia de peso, no se presentó diferencias significativas $(p>$ $0,05)$, por efecto de los tratamientos obteniendo una media de T2 (10\% de harina de totorilla) con 0,67 kg, siendo numéricamente el mejor valor seguido por T1 y T3 con $0,61 \mathrm{~kg}$ y los niveles T0 y T4 con 0,59 kg que presenta resultados más bajos.

La ganancia de peso de los animales según [7], al evaluar el efecto de diferentes niveles de harina de retama más melaza en la elaboración de bloques nutricionales en la alimentación de cuyes donde el investigador observó una ganancia de peso 


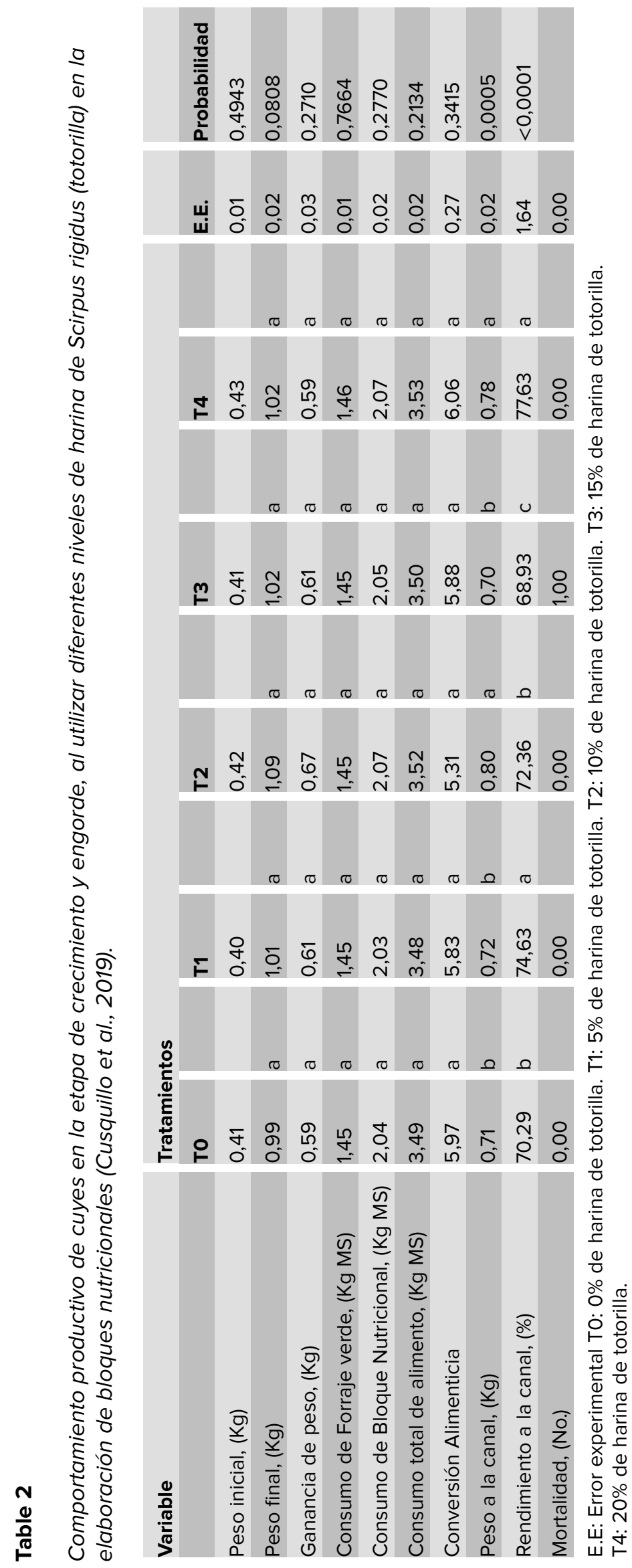


registrado de 0,566 kg. Mientras que [10], evaluó la aceptabilidad del bagazo de caña, rastrojo de maíz, tamo de cebada en bloques nutricionales como remplazo de maíz, siendo con la harina de bagazo de caña que se obtuvo mejor ganancia de peso con 0,566 kg. En cambio [9], estudió la utilización de bloques nutricionales a base de harina de maralfalfa en la alimentación de cuyes en la etapa de crecimiento y engorde, donde se han reportado una ganancia de peso $0,63 \mathrm{~kg}$ con un $30 \%$ de harina de maralfalfa. La variación del comportamiento ganancia de peso en los animales durante la etapa de crecimiento y engorde está determinada, por la eficiencia alimenticia que tiene relación con la energía y proteína al no contar con las cantidades necesarias resulta deficiente y no se obtiene resultados positivos [5]. Esto debido a que es un alimento de inmediata disponibilidad en el aparato digestivo de los animales, con mayor facilidad de absorción de sus componentes nutritivos [11].

\subsubsection{Consumo de forraje, $\mathrm{Kg}$ MS}

Al analizar los resultados de la variable consumo de forraje, no presentó diferencias significativas $(p>0,05)$, por efecto de los tratamientos obteniendo una media de 1,45 kg (T0); 1,45 kg (T1); 1,45 kg (T2); 1,45 kg (T3); y 1,46 kg (T4), siendo este último mayor al resto de tratamientos.

\subsubsection{Consumo de bloque, $\mathrm{Kg}$ MS}

Al analizar la variable consumo de bloque, no se presentó diferencias significativas ( $p$ $>0,05)$, por efecto de los tratamientos obteniendo una media del tratamiento en T2 ( $10 \%$ de harina de totorilla) y T4 ( $20 \%$ de harina de totorilla) con $2,07 \mathrm{~kg}$, cada uno, T1 con $2,03 \mathrm{~kg}$ que presenta consumo más bajo de los tratamientos.

\subsubsection{Consumo total de alimento, $\mathrm{Kg}$ MS}

Al analizar la variable consumo total de alimento, no se presentó diferencias significativas $(p>0,05)$, por efecto de los tratamientos obteniendo una media de los tratamientos en T4 ( $20 \%$ de harina de totorilla) y T2 (10\% de harina de totorilla) con 3,53 kg; 3,52 $\mathrm{kg}$ respectivamente, $\mathrm{T} 1 \mathrm{con} 3,48 \mathrm{~kg}$ que presenta menor consumo frente al resto de tratamientos.

Al comparar la variable consumo total de alimento [7], al evaluar el efecto de diferentes niveles de harina de retama más melaza en la elaboración de bloques nutricionales en la alimentación de cuyes en crecimiento y engorde, el consumo registrado fue de 4,572 kg. Mientras que [8], valoró diferentes niveles de harina de fideo en la alimentación de cuyes en la etapa de crecimiento y engorde en relación con el consumo registrado fue de 4,410 kg. En cambio [9], estudió la utilización de bloques nutricionales a base de harina de maralfalfa en la alimentación de cuyes en la etapa de crecimiento y engorde, reportando un consumo de $5,08 \mathrm{~kg}$ en el tratamiento $10 \%$ de harina de maralfalfa siendo superiores a los que se obtuvo dentro de nuestra investigación. La utilización de una ración mixta en la alimentación puede determinar el nivel y calidad 
de materia prima que se utilizada, y por lo tanto puede ser una determinante de la cantidad consumida por el animal, ya que a mejor calidad existirá un menor consumo [12].

\subsubsection{Conversión alimenticia}

Al analizar la variable conversión alimenticia, no se presentó diferencias significativas ( $p$ $>0,05)$, por efecto de los tratamientos obteniendo una media de los tratamientos en T2 (10\% de harina de totorilla) con 5,31, seguido por T1 y T3 con 5,83 y 5,88 respectivamente y T4 con 6,06.

Al analizar la variable conversión alimenticia según [7], que evaluó el efecto de diferentes niveles de harina de retama más melaza en la elaboración de bloques nutricionales en la alimentación de cuyes en crecimiento y engorde, conversión registrada fue de 8,12. Así también [10], al evaluar la aceptabilidad del bagazo de caña, rastrojo de maíz, tamo de cebada en bloques nutricionales como remplazo de maíz, siendo con la harina de bagazo de rastrojo de maíz que se obtuvo mejor conversión con 11,70. En cambio [9], al estudiar la utilización de bloques nutricionales a base de harina de maralfalfa en la alimentación de cuyes en la etapa de crecimiento y engorde, reportando un conversión de 8,06. Hace referencia a la eficiencia de asimilación de los animales hacia el alimento en nuestra investigación fue más eficiente.

\subsubsection{Peso a la canal, $\mathrm{Kg}$}

Al analizar la variable peso a la canal se obtuvieron diferencias altamente significativas $(p \leq 0,01)$, siendo el mayor peso para el T2 (10\% de harina de totorilla) con $0,80 \mathrm{~kg} \pm$ 0,02 , seguido por el T4 y T1 con 0,78 y 0,72 kg $\pm 0,03$ respectivamente, a continuación, el T0 y T3 con 0,71 y $0,70 \mathrm{~kg} \pm 0,02$ que presentaron resultados más menores.

Al analizar la variable peso a la canal [7], que evaluó el efecto de diferentes niveles de harina de retama más melaza en la elaboración de bloques nutricionales en la alimentación de cuyes en crecimiento y engorde, peso registrado fue de 0,67 kg. En cambio [9], al estudiar la utilización de bloques nutricionales a base de harina de maralfalfa en la alimentación de cuyes en la etapa de crecimiento y engorde, reportando un peso de $0,75 \mathrm{~kg}$. El peso a la canal se ve influenciada por la diferencia en el crecimiento de sus vísceras entre los animales, así como el tamaño de los testículos puede variar entre machos.

\subsubsection{Rendimiento a la canal, $\%$}

Al analizar la variable rendimiento a la canal se reportan diferencias altamente significativas $(p \leq 0,01)$, obteniendo los valores más altos para los tratamientos T4; T1; T2; TO con 77,63; 74,63; 72,36; 70,29 (\%) y se observó un menor valor en el T3 con 68,93\%.

Al analizar la variable rendimiento a la canal según [7], que evaluó el efecto de diferentes niveles de harina de retama más melaza en la elaboración de bloques 
nutricionales en la alimentación de cuyes en crecimiento y engorde, rendimiento al canal registrado fue de $69,67 \%$. Así también [10], al evaluar la aceptabilidad del bagazo de caña, rastrojo de maíz, tamo de cebada en bloques nutricionales como remplazo de maíz, siendo con la harina de bagazo de rastrojo de maíz que se obtuvo mejor rendimiento de la carcasa con $76,18 \%$. Mientras que [8], valoró diferentes niveles de harina de fideo en la alimentación de cuyes en la etapa de crecimiento y engorde en relación con la variable rendimiento de $67,77 \%$. En cambio [9], al estudiar la utilización de bloques nutricionales a base de harina de maralfalfa en la alimentación de cuyes en la etapa de crecimiento y engorde, reportando un rendimiento a la canal de 65,21\%. Siendo mayor los resultados que obtuvimos, esto puede deberse al comportamiento final que tienen los semovientes al momento del sacrificio, la misma que se debe realizar cuando el animal este en ayunas, la variación estará determinada directamente con su comportamiento [13].

\subsection{Comportamiento productivo de los cuyes en base al sexo}

Al evaluar el comportamiento productivo en base al factor sexo, no se encontraron diferencias estadísticas ( $p>0,05)$, en las variables: Consumo de forraje verde, consumo de bloque nutricional y consumo total de alimento, Tabla 3.

Mientras que, en las variables: Peso final, ganancia de peso, conversión alimenticia, peso a la canal y rendimiento a la canal se presentaron diferencia significativa $(p \leq$ $0,05)$, siendo los machos los que presentaron los mejores resultados con relación a las cuyas hembras durante el desarrollo de la investigación. Mientras tanto en las variables: consumo de forraje verde, consumo del bloque nutricional y el consumo total de alimento (kg MS), no se registraron diferencias significativas.

\section{Table 3}

Comportamiento productivo de cuyes en la etapa de crecimiento y engorde, en base al factor sexo (Cusquillo et al., 2019).

\begin{tabular}{l|l|l|l|l|l|l} 
& Machos & & Hembras & & \\
Variable & & & & & E.E* & Probabilidad \\
\hline Peso inicial, (Kg) & 0,42 & & 0,41 & & 0,01 & 0,37010 \\
\hline Peso final, (Kg) & 1,06 & a & 0,99 & b & 0,01 & 0,00250 \\
\hline Ganancia de peso, (Kg) & 0,64 & a & 0,58 & b & 0,02 & 0,02390 \\
\hline Consumo de Forraje verde, (Kg MS) & 1,46 & a & 1,45 & a & 0,005 & 0,49740 \\
\hline Consumo de Bloque Nutricional, (Kg MS) & 2,06 & a & 2,04 & a & 0,01 & 0,39680 \\
\hline Consumo total de alimento, (Kg MS) & 3,51 & a & 3,49 & a & 0,01 & 0,28420 \\
\hline Conversión Alimenticia & 5,55 & a & 6,07 & b & 0,17 & 0,04300 \\
\hline Peso a la canal, (Kg) & 0,77 & a & 0,72 & b & 0,01 & 0,00190 \\
\hline Rendimiento a la canal, (\%) & 75,98 & a & 69,56 & b & 1,04 & 0,00570 \\
\hline Mortalidad, (No.) & 0,00 & & 1,00 & & 0,00 &
\end{tabular}

E.E.: Error estándar.

Prob. > 0,05: No existen diferencias estadísticas; Prob. < 0,05: Existen diferencias estadísticas; Prob. < 0,01 : Existen diferencias altamente significativas. 


\subsection{Comportamiento de la interacción de cuyes alimentados con diferentes niveles de harina de totorilla frente al sexo}

Al analizar la interacción se observa que en la variable peso a la canal kg, presenta diferencias altamente significativas $(p \leq 0,01)$, con valor más alto en machos de tratamiento $10 \%$ con $0,82 \mathrm{~kg}$ y el más bajo en hembras del tratamiento $5 \%$ con 0,67 kg. En la variable rendimiento a la canal \%, existen diferencias altamente significativas $(p \leq 0,01)$, entre los tratamientos en estudio, observándose que los animales machos faenados de T4, son los mejores con $80,87 \%$ y los animales machos del T0 son las que presentaron rendimiento más bajo con $67,49 \%$.

\subsection{Mortalidad, No.}

En relación con la mortalidad al adicionar diferentes niveles de harina de totorilla en la alimentación de cuyes en crecimiento y engorde no influyo estadísticamente, por lo cual las respuestas de mortalidad registrados fueron de 1 animal hembra del tratamiento con $15 \%$ de harina de totorilla, cuya causa se debió a problemas del manejo general.

\subsection{Análisis bromatológico de los bloques}

El análisis bromatológico del bloque nutricional a base de harina de totorilla, realizado en los laboratorios de AGROLAB, las mismas que arrojaron las siguientes respuestas que se detallan en la Tabla 5.

\subsubsection{Proteína, \%}

Al evaluar el porcentaje de proteína de los bloques en base a harina de totorilla, en la presente investigación reporta un promedio en el T0 de 7,48\%; T1 8,82\%; T2 9,22\%; T3 10,83\%; T1 11,23\%.

\subsubsection{Materia seca, $\%$}

Al evaluar el porcentaje de materia seca de los bloques en base a harina de totorilla, en la presente investigación reporta un promedio en el T0 de 91,99\%; T1 88,16\%; T2 87,59\%; T3 90,89\%; T4 89,00\%.

\subsubsection{Humedad, \%}

Al evaluar el porcentaje de humedad de los bloques en base a harina de totorilla, en la presente investigación reporta un promedio en el T0 de 8,01\%; T1 11,84\%; T2 12,41\%; T3 9,11\%; T4 11,00\%. 


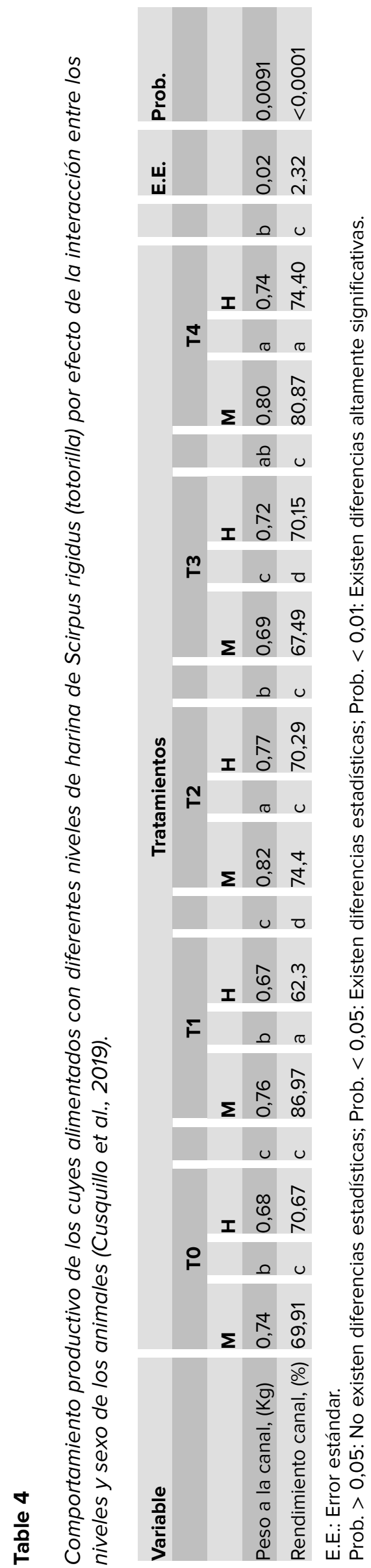




\section{Table 5}

Análisis bromatológico de los bloques nutricionales a base de harina de totorilla (AGROLAB, 2020).

\begin{tabular}{l|l|l|l|l|l|l}
\hline & \multicolumn{7}{c}{ Resultados } & & & \\
Parámetro & Unidad & T0 & T1 & T2 & T3 & T4 \\
\hline Proteína & $\%$ & 7,48 & 8,82 & 9,22 & 10,83 & 11,23 \\
\hline Materia seca & $\%$ & 91,99 & 88,16 & 87,59 & 90,89 & 89,00 \\
\hline Humedad & $\%$ & 8,01 & 11,84 & 12,41 & 9,11 & 11,00 \\
\hline Grasa & $\%$ & 6,82 & 7,42 & 8,13 & 8,83 & 8,03 \\
\hline Fibra & $\%$ & 9,90 & 10,15 & 10,73 & 11,49 & 12,48 \\
\hline Cenizas & $\%$ & 6,53 & 7,32 & 8,50 & 10,82 & 12,39 \\
\hline Extracto libre de nitrógeno & $\%$ & 61,27 & 54,46 & 51,01 & 48,92 & 44,87
\end{tabular}

\subsubsection{Grasa, \%}

Al evaluar el porcentaje de grasa de los bloques en base a harina de totorilla, en la presente investigación reporta un promedio en el T0 6,82\%; T1 7,42\%; T2 8,13\%; T3 8,83\%; T4 8,03\%.

\subsubsection{Fibra, \%}

Al evaluar el porcentaje de fibra de los bloques en base a harina de totorilla, en la presente investigación reporta un promedio en el T0 9,90\%; T1 10,15\%; T2 10,73\%; T3 11,49\%; T4 12,48\%. Los porcentajes de este elemento en balaceados utilizados para la alimentación de cuyes varían del 5 al 18\%. Este componente tiene importancia en la composición de la dieta ya que favorece la digestibilidad de otros nutrientes, pues retarda el paso del contenido alimenticio a través del aparato digestivo [4]. El contenido de fibra en la composición de las raciones es necesario por la capacidad que tienen los cuyes de digerirla, además retarda el pasaje del contenido alimenticio [14].

\subsubsection{Cenizas, \%}

Al evaluar el porcentaje de cenizas de los bloques en base a harina de totorilla, en la presente investigación reporta un promedio en el T0 6,53\%; T1 7,32\%; T2 8,50\%; T3 10,82\%; T4 12,39\%. Siendo los principales formadores de los huesos y los dientes, y un 3 a $4.5 \%$ del peso del cuerpo [1]. En cuyes el consumo de minerales para su crecimiento y engorde es de 1,20 por ciento de calcio y 0.6 por ciento de fósforo, para evitar problemas de orden metabólico [2, 4].

\subsubsection{Extracto libre de nitrógeno, \%}

Al evaluar el porcentaje de extracto libre de nitrógeno de los bloques en base a harina de totorilla, en la presente investigación reporta un promedio en el T0 61,27\%; T1 
54,46\%; T2 51,01\%; T3 48,92\%; T4 44,87\%. ELN (extracto libre de nitrógeno), representa a la fracción de los carbohidratos solubles es una mezcla de almidones, azucares, hemicelulosa y lignina, puede contener además vitaminas hidrosolubles, no obstante, la mayor parte del ELN se compone de almidón y azúcares (alto valor energético) [15].

\subsection{Relación beneficio/costo, $\$$}

Al realizar la evaluación económica en la alimentación de cuyes con los diferentes niveles de harina de totorilla durante la etapa de crecimiento y engorde, se determinó que el mejor beneficio/costo fue mejor para el tratamiento con $20 \%$ de harina de totorilla con 1,32 USD, lo que significa que, por cada dólar invertido se obtiene una ganancia de 0,32 USD, mientras que el menor beneficio/costo se registró en tratamiento con 0\% con harina de totorilla con 1,29 USD.

\section{Conclusiones y Recomendaciones}

Los resultados obtenidos en el presente trabajo experimental damos a conocer en las siguientes conclusiones:

1. Los resultados reportados muestran que el uso de harina de totorilla en la alimentación de cuyes en la etapa de crecimiento y engorde en forma de bloque nutricional no afectó los parámetros productivos y la salud de los animales.

2. Al evaluar el comportamiento productivo de los cuyes alimentados con diferentes niveles de harina de totorilla, en las variables peso final, ganancia de peso, consumo de alimento, conversión alimenticia, no reportaron diferencias estadísticas entre los tratamientos, únicamente se reportaron diferencias altamente significativas $(p \leq 0,01)$, en la variable peso a la canal y rendimiento a la canal.

3. En lo que al factor sexo se refiere, influye de forma relevante en el rendimiento productivo, pues los resultados demuestran que al utilizar los diferentes niveles de harina de totorilla se pudo observar que las variables: peso final, ganancia de peso, conversión alimenticia, peso a la canal y rendimiento a la canal se presentaron diferencias significativas ( $p \leq 0,01)$, a favor de los machos, con respecto a las cuyas hembras.

4. El mejor beneficio/costo se reportó en el tratamiento con $20 \%$ de harina de totorilla con 1,32 USD, lo que significa que, por cada dólar invertido en la producción, se obtiene una ganancia de 0,32 USD, mientras que el beneficio/costo más bajo se determinó en el nivel 0\% con harina de totorilla con 1,29 USD.

De acuerdo con los resultados obtenidos se pueden determinar las siguientes recomendaciones:

1. Realizar nuevas investigaciones con estos semovientes, pero en otras etapas fisiológicas como: Gestación y lactancia, utilizando los mismos niveles de harina de totorilla, para observar el comportamiento reproductivo. 
2. Realizar nuevas investigaciones, pero en otras especies de interés zootécnico (conejos, ovinos, caprinos) durante las diferentes etapas fisiológicas utilizando la harina de totorilla para su alimentación diaria.

3. Difundir los resultados alcanzados en la presente investigación a los pequeños, medianos y grandes productores de cuyes con la finalidad de abaratar los costos de producción mediante la implementación del uso de la harina de totorilla en la alimentación de estos semovientes.

\section{References}

[1] Zeas-Delgado V. Análisis productivo, indice de conversión y mortalidad en cuyes durante el periodo de engorde, manejados en pozas y jaulas [Tesis pregrado]. Cuenca: UPS; 2011.

[2] Sihuacolla-Mamani E. Influencia de ración balanceada en pellets sobre la ganancia de peso vivo en cuyes (Cavia porcellus L.) [Tesis Pregrado]. Puno: UNAP; 2013.

[3] Marchan-Valencia K. Efecto del uso de los manano-oligosacáridos en la dieta de cuyes (Cavia porcellus) en la fase de crecimiento-engorde sobre el comportamiento productivo y rentabilidad económica [Tesis pregrado]. Trujillo: UPAO; 2019.

[4] Chauca L. Producción de cuyes (Cavia porcellus) nutrición y alimentación. Lima; 1997. Disponible en: https://books.google.es/books?hl=es\&lr=\&id=VxLVzsZ5HWcC\&oi=fnd\&pg=PP9\&dq= liliana+chauca+produccion+de+cuyes.

[5] Narváez-Sarango J. Ritmo de cecotrofia en cuyes (Cavia porcellus) [Tesis pregrado]. Loja: UNL; 2018.

[6] Sandoval-Alarcón H. Evaluación de diferentes tipos de dietas en cobayos en crecimiento [Tesis pregrado]. Ambato: UTA; 2013.

[7] Quinatoa-Quiquintuña S. Evaluación de diferentes niveles de harina de retama más melaza en la elaboración de bloques nutricionales para la alimentación de cuyes [Tesis Pregrado]. Riobamba: ESPOCH; 2007.

[8] Silva M. Evaluación del efecto de tres niveles de harina de fideo (10, 20 y 30 \%) en la alimentación de cuyes mejorados durante el crecimiento y engorde [Tesis pregrado]. Loja: UNL; 2013.

[9] Gualoto-Lata G. Evaluación de diferentes niveles de harina de Pennisetum violaceum (maralfalfa) en la elaboración de bloques nutricionales y su utilización en la alimentación de cuyes en la etapa de crecimiento y engorde [Tesis pregrado]. Riobamba: ESPOCH; 2018.

[10] Imba E, Tallana L. Aceptabilidad del bagazo de caña, rastrojo de maíz y tamo de cebada en bloques nutricionales como reemplazo del maíz en cobayos de engorde (Cavia porcellus) en la granja la PraderaChaltura [Tesis pregrado]. Ibarra: UTN; 2011.

[11] Castillo $\mathrm{C}$ et al. Efecto de la suplementación con bloques minerales sobre la productividad de cuyes alimentados con forraje. Revista de Investigación Veterinaria. 2012.

[12] Carlos-Díaz L. Combinacion de pasto vara San Jose (Scirpus maritimus L.) con maiz chala (Zea mays) en alimentacion de cuyes en engorde en la provincia de Chiclayo - Lambayeque [Tesis pregrado]. Lambayeque: UNPRG; 2015.

[13] Carabajal-Chávez C. Evaluación preliminar de tres alimentos balanceados para cuyes (Cavia porcellus) en acabado en el Valle del Mantaro [Tesis pregrado]. Lima: UNALM; 2015.

[14] Ambuludí-Sarango L. Evaluación de la achupalla (Puya eryngioides) en la alimentación de cuyes mejorados en el cantón Saraguro [Tesis pregrado]. Loja: UNL; 2011.

[15] Vilchez A, VergaraV. Evaluación de diferentes densidades de nutrientes en dietas con exclusión de forraje para cuyes en crecimiento en condiciones de verano de la costa central del Perú [Tesis pregrado]. Lima: UNALM; 2014.

[16] Tapia M. Pasto naturales del altiplano de Perú y Bolivia. Quito: Instituto Interamericano de Ciencias Agricolas; 1971. pp. 32-100.

[17] Salazar-Espinoza D. Análisis químico y digestabilidad in vitro de cinco especies forrajeras nativas recolectadas en dos épocas del año [Tesis pregrado]. La Paz: UMSA; 2006.

[18] Esquivel-Valverde V. Bloques nutricionales. 2011. Disponible en: http://www.mag.go.cr/bibliotecavirtual/ dr-brunca-boletin-inf-asa-neily-junio-2011.pdf

[19] Paco-Tintaya S. Efecto de cuatro niveles de totora (Shoenoplectus californicus) en el engorde en cuyes (Cavia porcellus) en la comunidad de Achocara, municipio de Luribay, provincia Loayza del departamento La Paz [Tesis pregrado]. La Paz: UMSA; 2016. 
[20] Mendoza A. Requerimientos de proteína del cuy. Fundamentos de nutrición y alimentación. Lime: Instituto Nacional de Investigación Agraria; 2012.

[21] Olmedo-Guaman S. Utilización de diferentes niveles de ensilaje de maiz en la alimentación de cuyes en la etapa de crecimiento y engorde [Tesis pregrado]. Riobamba: ESPOCH; 2015.

[22] Organización de las naciones unidas para la agricultura y la alimentaria. 2005. Disponible en; http: //www.fao.org/3/a-at783s.pdf.

[23] Paucar-Paucar D. Evaluaciòn del efecto del uso de bloques nutricionales como dieta suplementaria en la alimentacion de cuyes destetados (Cavia porcellus) [Tesis pregrado]. Ambato: UTA; 2013.

[24] Perez-Shuña L. Efecto de la alimentación con Erythrina sp vs Pueraria phaseloides en cuyes criollos (Cavia porcellus) sobre parametros productivos [Tesis pregrado]. Yurimaguas-Loreto: UNAP; 2013. 